\title{
THE INTERGALACTIC MEDIUM
}

Dr. George B. Field

Harvard-Smithsonian Center for Astrophysics

Observations of Lyman- $\alpha$ absorption show that any gas present in the space between clusters of galaxies must be hot, and $x$-ray observations indicate that there may be present gas whose temperature at low redshifts is $4 \times 10^{8} \mathrm{~K}$ and whose density is about half the critical value for cosmology. The interpretation of these observations is reviewed, and difficulties with proposed heating mechanisms are emphasized.

\section{INTRODUCTION}

Presumably the intergalactic medium (IGM), if it exists at all, is the lowest-density gas in the universe. From Friedmann's general relativistic models of the expanding universe we know that a density

$$
\rho_{c}=\frac{3 \mathrm{Ho}^{2}}{8 \pi \mathrm{G}}=4.7 \times 10^{-30} \mathrm{~g} \mathrm{~cm}^{-3}
$$

for the Hubble constant $\mathrm{H}_{O}=50 \mathrm{~km} \mathrm{sec}^{-1} \mathrm{Mpc}^{-1}$ is critical in the sense that a universe with a greater density will ultimately collapse under its own self gravitation, while one with less will expand forever. observations of galaxies indicate that their contribution to the smoothed-out density of matter in the universe is given by

$$
\Omega_{\mathrm{GAL}} \equiv \frac{\left\langle\rho_{\mathrm{GAL}}\right\rangle}{\rho_{\mathrm{C}}} \simeq 0.05
$$

(Gott, et al. 1974), so that if most of the matter is in galaxies, the universe is underdense. This accords with the observations of the abundance of intersteliar ${ }^{2} \mathrm{H}$, which indicate that $\Omega$ including all forms of normal matter must be $\lesssim 0.1$ if the ${ }^{2} \mathrm{H}$ was produced in the big bang (Yang, et al. 1979).

The smoothed-out density of protons corresponding to $\Omega=1$ is about $2 \times 10^{-6} \mathrm{~cm}^{-3}$ (if $\mathrm{He} / \mathrm{H}=0.1$ ). Hence there has long been interest in whether intergalactic space contains this much gas. The

Patrick A. Wayman (ed.), Highlights of Astronomy, Vol. 5, 375-386.

Copyright (c) 1980 by the IAU. 


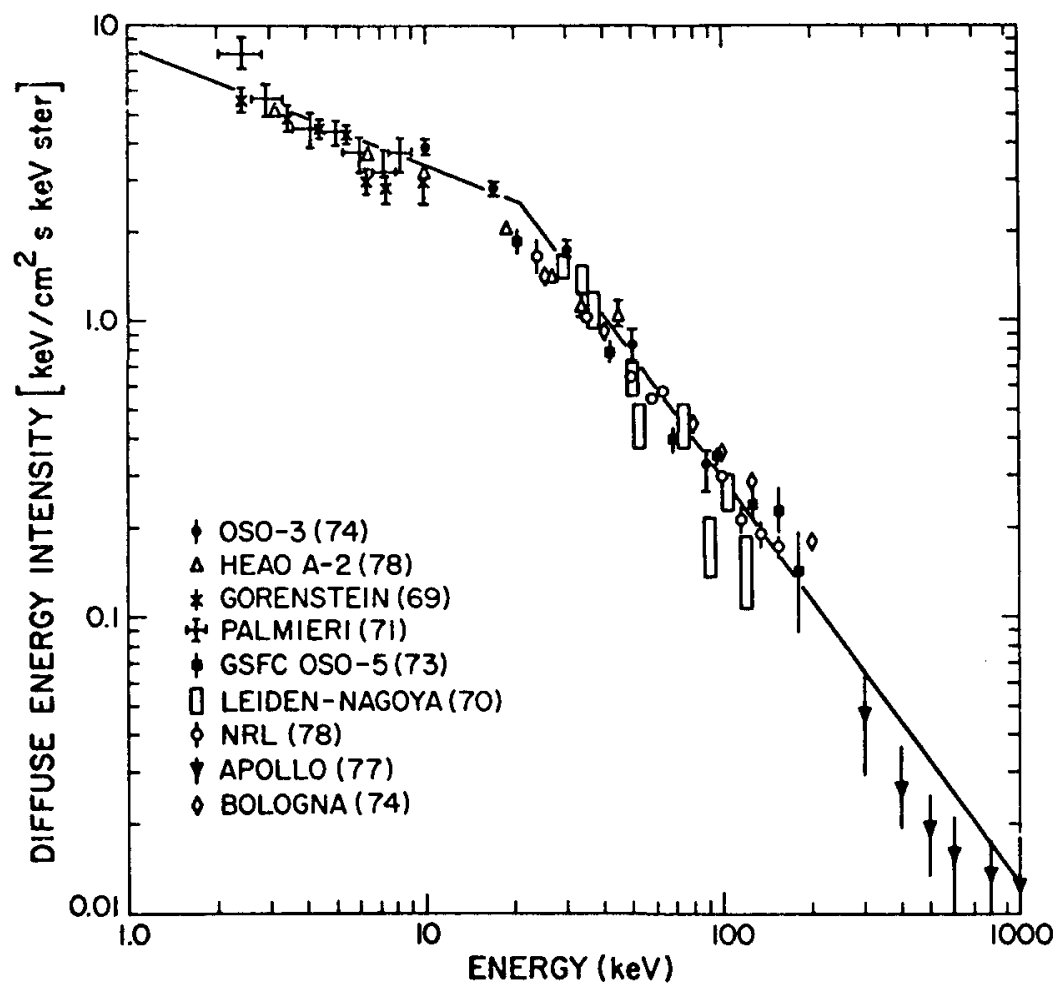

Figure 1.

The mean intensity of the $x$-ray background over the energy range 1 to $1000 \mathrm{keV}$, compiled from a number of experiments in different years. The broken power law represented by the two straight lines is one representation of the data, but not unique (Schwartz 1979). 
lack of a strong Lyman - $\alpha$ absorption trough in the spectra of quasars puts severe limits on the density of neutral gas, so that any gas present must be highly ionized. In view of the hot gas in galaxy clusters discovered by $x$-ray astronomers (Culhane, the previous paper) it seems natural to suppose that the IGM(by which we mean the gas which occupies the great majority of intergalactic space outside of galaxy clusters) is also very hot. Speculation that this is the case, and indeed that the density of IGM is significant in the sense that

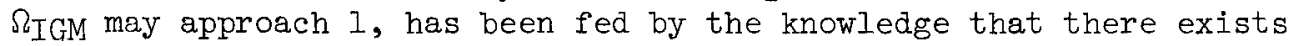
a nearly isotropic $x$-ray background whose spectrum can be fit over a large energy range ( $\sim$ to $100 \mathrm{keV}$ ) by a thermal bremsstrahlung (TB) spectrum with $\mathrm{kT} \simeq 45 \mathrm{keV}\left(\mathrm{T} \simeq 5.4 \times 10^{8} \mathrm{~K}\right)$. This is what would be predicted if there existed a smoothly-distributed IGM which had been heated by some unknown process (Cowsik and Kobetich 1972). In what follows we discuss whether this simple interpretation is likely to be correct.

\section{OBSERVATIONS}

Two types of observations of the $x$-ray background have been carried out. Figure 1 shows the spectrum of the mean intensity as derived from many experiments over a large energy range, while Figure 2 shows the spectrum over the smaller range 3 to $50 \mathrm{keV}$ derived from a precise experiment on HEAO-1. As can be seen, the fit to a TB spectrum is quite good over the range 1 to $100 \mathrm{keV}$, and the fit over the narrower range is excellent. The derived temperatures are so high $\left(\sim 5 \times 10^{8} \mathrm{~K}\right)$ that no lines are expected, and none is seen. The lack of lines prevents us from carrying out the spectroscopic diagnostics described earlier in this Joint Discussion.

In the energy range below $1 \mathrm{keV}$, the intensity rises above the $5 \times 10^{8} \mathrm{~K} T B$ curve. Because the radiation is increasingly observed to be associated with the Milky Way as the photon energy is reduced, this effect is attributed to hot gas within our galaxy, as described above by Cox. In the energy range above $100 \mathrm{keV}$, the intensity does not fall as rapidly as would be predicted by the TB interpretation. This could be used as an argument against that interpretation, or, on the other hand, it may simply be the result of the contributions of additional sources having harder spectra.

It is the high degree of isotropy which led to the suggestion that the $x$-ray background above $1 \mathrm{keV}$ is cosmological in nature. Since we know that extragalactic $x-r a y$ sources exist (galaxies, quasars, clusters of galaxies) the radiation is not perfectly isotropic. However, it becomes more so as fainter and fainter sources are excluded from calculating the point-to-point variation.

Information on this point is available from the deep survey conducted with HEAO-2 in the range 1 to $4 \mathrm{keV}$. According to Giacconi, et al. (1979), the number of faint sources, believed to be largely 


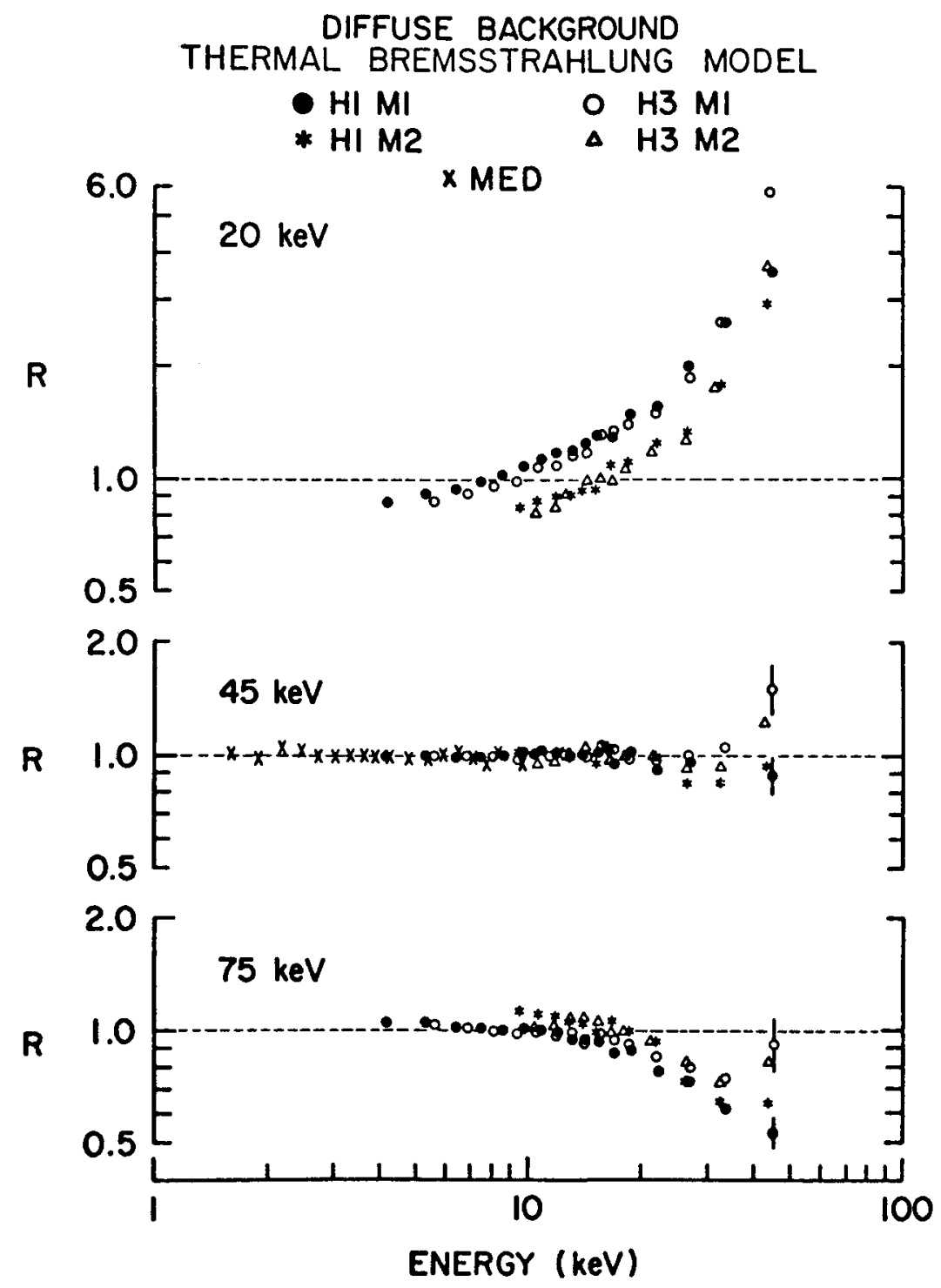

Figure 2.

The mean intensity of the $x$-ray background from a precise experiment on HEAO-1, represented by the ratio, $R$, of the mean intensity to that calculated for a single-temperature thermal bremsstrahlung emission. The fit is best for $\mathrm{kT}=45 \mathrm{keV}$. (Marshal1 et al. 1979). 
quasars, is so great that virtually all the $x$-ray background in the 1 - $4 \mathrm{keV}$ energy band can be accounted for, leaving no room for a contribution by the IGM. However, the data are not yet statistically precise enough to state this with absolute certainty, and there remains the question of whether this explanation applies to the entire $1-100$ $\mathrm{keV}$ range under discussion. Answering this question is an important future goal for observational x-ray astronomy.

In view of the apparent contradiction posed by the HEAO-I and HEAO-2 observations, it would be desirable if there were further tests of the TB hypothesis. One was pointed out by E. Purcell; just as in the case of intracluster gas, a hot IGM should scatter the cosmic microwave background ( $\mathrm{CMB}$ ), distorting its spectrum slightly as a result of the Compton effect ( ince $\mathrm{kT} / \mathrm{mc}^{2} \simeq 0.1$ ). This effect was calculated by Field and Perrenod (1977) on the basis of a nonrelativistic diffusion approximation. Wright (1979) correctly pointed out that the relevant optical depth is too small and the temperature is too high for this calculation to be valid; his results (Figure 3 ) indicate that the effect is too small to be detected by presently available techniques. However, it is hoped that future satellite experiments of much greater precision will test it.

\section{HEATING AND COOLING}

As shown by Field and Perrenod (1977), the main cooling effects are the cosmological expansion (if the gas is not gravitationally bound to any system), Compton cooling via collisions of thermal electrons with low-energy photons of the CMB, and, possibly, thermal conduction into any cooler gas which may be present (e.g., in galaxies or clusters of galaxies); radiation is negligible, as can be seen from the fact that the energy density of the $\mathrm{x}$-ray background is only $\sim 10^{-3}$ of that of the hot gas.

To proceed we must use the concept of redshift. In a Friedmann model the distance between two gravitationally unbound galaxies is proportional to a time-dependent scale factor $R(t)$, whose time dependence follows from Einstein's equations, once it has been decided whether the universe is open $(\Omega \leq 1)$ or closed $(\Omega>1)$. The wavelength of a photon measured at time $t$ is proportional to $R(t)$; the light emitted by galaxies is constantly shifting to longer wavelengths as the universe expands. Between the time a photon is emitted and the time it is observed, its wavelength increases by an amount (called the redshift)

$$
\mathrm{z} \equiv \frac{\lambda_{0 b s}-\lambda_{e m}}{\lambda_{\text {em }}}=\frac{R_{0 b s}-R_{e m}}{R_{e m}}
$$

Thus, $1+z$ calculated from $\lambda_{\text {obs }}$ for some spectral feature of an object is the ratio of the size of the universe now to that when the light was emitted by the object. 


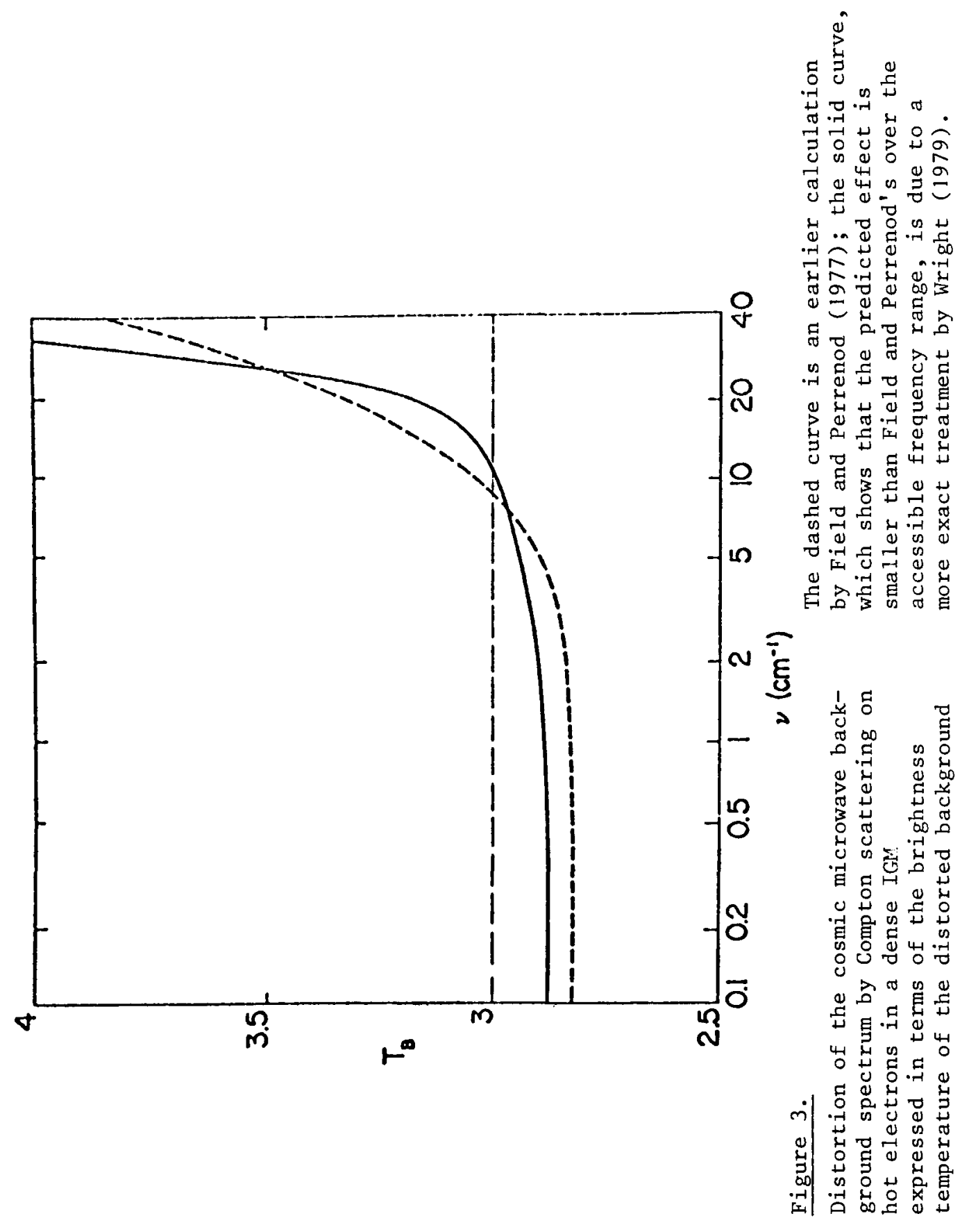


Field and Perrenod (1977) show that for redshifts $\leq 3$ (i.e., Rem $\left.>\frac{1}{4} R_{\text {obs }}\right)$, cooling is dominated by the cosmological expansion, which for a constant-entropy gas gives

$$
\mathrm{T} \sim \mathrm{R}^{-2} \sim(1+z)^{2}
$$

Compton cooling is less than 13\%, and conduction cooling must be negligible because otherwise the x-ray background from the gas where the energy is ultimately radiated would greatly exceed that observed.

The heating of a putative intergalactic medium poses a serious problem. At a present $(z=0)$ temperature of $5 \times 10^{8} \mathrm{~K}$, the energy content is $10^{17} \mathrm{erg} \mathrm{g}^{-1}$, or, for $\Omega_{\mathrm{IGM}}=0.5\left(\rho_{\mathrm{IGM}}=2.4 \times 10^{-30} \mathrm{~g} \mathrm{~cm}^{-3}\right)$, $2.4 \times 10^{-13} \mathrm{erg} \mathrm{cm}^{-3}$ (about $0.15 \mathrm{eV} \mathrm{cm}^{-3}$ ). This is about half of the energy contained in the CMB, heretofore by far the largest energy reservoir in the universe. Suppose this energy ultimately originates in galaxies, for which $\Omega_{\mathrm{GAL}} \simeq 0.05$. Since $\Omega_{\mathrm{IGM}}$ would be 10 times larger, the energy required from each gram of galactic matter would be $10^{18}$ erg $g^{-1}$, roughly equal to the energy released from an evolving star by producing helium. The problem here is that we know that the great bulk of the energy from stars is emitted in photons near $1 \mathrm{eV}$, which are totally unable to heat a gas to $50 \mathrm{keV}$. If galaxies are ultimately responsible, the energy must be emitted in some nonthermal form, such as fast particles or coherent waves. This suggests that the energy source responsible for heating the IGM is quasar outbursts.

Field and Perrenod (1977) evaluated quasars as an energy source, taking into account that the frequency of quasars was much higher in the past. If it is assumed that the frequency of quasars increases like $(1+z)^{6}$ back to the time $z=3$, falling rapidly at larger $z$, one obtains the time dependence of the gas temperature shown in Figure 4 ; a period of rapid heating ensues as quasars turn on at $z \simeq 3$, but for redshifts smaller than 2, adiabatic expansion losses dominate.

The temperature scale of Figure 4 is established by the energy delivered per quasar outburst, or, what amounts to the same thing, the energy, $E$, delivered in quasar outbursts per average bright galaxy, integrated over its lifetime. Field and Perrenod adjusted the temperature scale to satisfy the shape of the observed $x$-ray spectrum (Figure 1), obtaining $\mathrm{T}(\mathrm{z}=0)=4.4 \times 10^{8} \mathrm{~K}$ and $\mathrm{E}=1.6 \times 10^{64} \mathrm{erg}$, or $\sim 9 \times 10^{18} \mathrm{erg} \mathrm{g}^{-1}$ for a typical bright galaxy weighing $9 \times 10^{11} \mathrm{M}_{\odot}$ (including dark halo material). The energy required from each gram of galactic matter is about 9 times the value estimated naively above, because of the adiabatic expansion losses since $z \simeq 3$. Since

$9 \times 10^{18} \mathrm{erg} \mathrm{g}^{-1}$ is $0.01 \mathrm{c}^{2}$, one is forced to consider galactic explosions of great magnitude, presumably leaving behind gravitationally collapsed objects such as massive black holes of $\sim 10^{10} \mathrm{M}_{\odot}$. Not only does this seem excessive, at least for a typical galaxy, but there is no evidence that the energy released in a given quasar outburst reaches anything like the $3 \times 10^{62}$ ergs required from quasar statistics and 


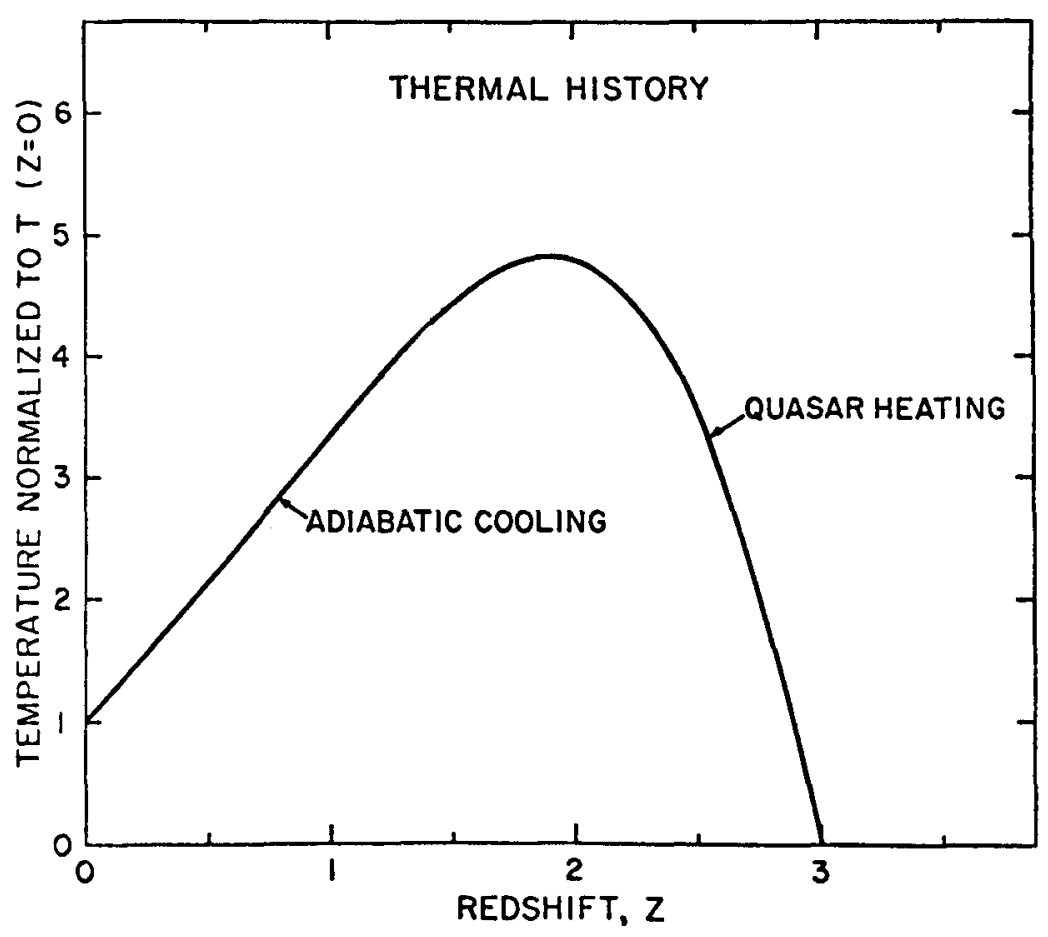

Figure 4.

The temperature of a uniform IGM calculated by Field and Perrenod (1977) for the case of heating by quasars assumed to turn on at redshift $z=3$ and to decline in numbers thereafter as $(1+z) 6$. The temperature at $z=0$ derived from the observed spectrum is $4.4 \times 10^{8} \mathrm{~K}$, so that the maximum temperature achieved is about $2 \times 10^{9} \mathrm{~K}$. 
ne assumption that the duration of each outburst is $10^{6}$ years. Thus, this model seems a priori unlikely. Moreover, Field (1979) has pointed out a more specific problem with quasar heating. The energy per bright galaxy, $10^{64} \mathrm{erg}$, is roughly equal to the energy by which intracluster gas is gravitationally bound into a cluster. Since there are 100 bright galaxies in a rich cluster like Coma (Abe11 1965), one would expect far more energy to be dumped in the intracluster gas than it could absorb and still stay bound. The only way to reconcile this with the observed presence of gas in clusters would be to assume that quasar outbursts are much more infrequent in cluster galaxies than in the field. What fragmentary evidence exists on this point actually indicates that quasars are more frequent in clusters (Field 1979). Thus, heating by quasars meets a fundamental objection.

Field (1979) considered an alternative model for heating suggested by Maraschi and Perola (1976): that very massive intergalactic clouds free fall and shock heat to temperatures of the order required without ever cooling and fragmenting significantly. Such a model would have certain advantages in accounting for the $x$-ray background. Its thermal spectrum would result if most clouds had about the same temperature. Because clumped gas is more effective, per gram, in radiating TB, $\Omega$ IGM could be reduced below 0.1 , as required by the observed abundance of ${ }^{\mathrm{H}}$. Finally, gravitation would be the ultimate source of the heat. Although this model is rather appealing, it founders on the observed high degree of isotropy of the background. According to Field (1979) the gravitationally-bound clouds of hot gas would result in a normalized point-to-point variance in intensity given by

$$
\frac{\left\langle(\delta \mathrm{I})^{2}\right\rangle}{\langle\mathrm{I}\rangle^{2}} \equiv \sigma^{2} \geq \frac{32}{9}\left(\frac{\mathrm{S}_{\mathrm{e}}}{\mathrm{S}_{\mathrm{o}}}\right)\left(\frac{4 \pi}{\mathrm{S}_{\mathrm{o}}}\right) \frac{\alpha_{\mathrm{IGM}}}{{ }^{\Omega_{\mathrm{IGM}}}}
$$

Here $\mathrm{S}_{e}$ is the effective solid angle of the detector for detection of point sources, $S_{0}$ is the total solid angle,

$$
\propto_{\mathrm{IGM}}=\frac{3 \mathrm{RT}}{2 \mu \mathrm{c}^{2}}=1.1 \times 10^{-4}
$$

for $\mathrm{T}=4.4 \times 10^{8} \mathrm{~K}$, and $\theta$ is the angular radius of the collimator. Since $\sigma=0.07$ is the observed value including all extragalactic sources, (Field 1979) equation (5) applied to UHURU implies that

$$
\Omega_{\text {IGM }} \geq 3
$$

This violates the fact that the mean $\mathrm{x}$-ray intensity $<\mathrm{I}>$ requires $\Omega_{I G M} \leq 0.5$. Since $\left\langle I>\right.$ is proportional to $\Omega_{I G M}$, equation (7) would imply an $x$-ray intensity more than 36 times IGMat observed. The reasoning behind equation (5) is that one can adjust the mass (and hence radius) of the putative clouds only within certain limits. If one increases the radius in order to make at least the nearer clouds resolvable (so they will not contribute to $\sigma^{2}$ ), the mass of each cloud, and hence $\Omega_{\text {IGM }}$, increases in proportion. If, 
on the other hand, one decreases the radius, all the clouds are unresolved and hence contribute to $\sigma^{2}$; the only way to decrease $\sigma^{2}$ is to increase the number density of clouds, and this again increases $\Omega$ IGM. I conclude that models based upon gravitationalclumping and shock heating are also unlikely to heat the IGM. At this time, I know of no reasonable way to do it; I am therefore skeptical about the existence of a hot dense IGM.

Before going on it is interesting to speculate upon the fact that the observed spectrum above lookeV falls less rapidly than the TB model. of course the reason that the TB spectrum falls is the absence of very energetic electrons $(E \gg \mathrm{kT}$ ) in a relativistic Maxwellian. But for the Maxwellian to apply, the electrons must suffer elastic collisions. Given that the elastic cross section drops rapidly at high energies, it could be that the number of energetic electrons above $\mathrm{kT}$, and consequently, the bremsstrahlung emission, is unexpectedly large. This situation is reminiscent of laboratory plasma experiments, where runaway electrons are observed whose velocities are so high that no collisions are likely in the duration of the experiment. By analogy once can calculate the energy which an electron would have now if it were injected into the IGM at high enough energy that it would probably not collide: the answer is $\mathrm{E} \simeq 100 \mathrm{keV}$. This lends plausibility to the suggestion that if the bulk of the $x$-ray background is $T B$, the tail above $100 \mathrm{keV}$ is due to bremsstrahlung by electrons which have not yet been thermalized since they were first accelerated.

\section{DYNAMICAL EFFECTS}

Suppose for the sake of discussion that a hot $\left(4.4 \times 70^{8} \mathrm{~K}\right)$, dense $(\Omega=0.5)$ IGM does exist. Its energy density is $2.4 \times 10^{-13} \mathrm{erg} \mathrm{\textrm {cm } ^ { - 3 }}$ and its pressure, $1.6 \times 10^{-13}$ dyne $\mathrm{cm}^{-}\left(\mathrm{p} / \mathrm{k}=1100 \mathrm{~cm}^{-3} \mathrm{~K}\right)$. This pressure is of the same order as exists in the ISM in our galaxy, recalling the suggestion by Gold and Hoyle (1959) of a universe in pressure equilibrium. Their notion was that if the IGM were hot ( $10^{9} \mathrm{~K}$ in their model, due to $B$ decay of recently created neutrons) "it is attractive to regard the galaxies as regions of local cooling in a hot gas. Condensation is not then primarily a gravitational process, but a result of pressure in the external hot gas acting at the surface of a cooled region."

For condensation to occur, there must be an energy flux, either radiative or conductive. Normally one can ignore heat conduction on scales like megaparsecs, but pogause the thermal conductivity increases rapidly with temperature $\left(\kappa^{\sim} \mathrm{T}^{5 / 2}\right)$, it would be important in the IGM provided that there is not a tangled magnetic field. Ultimately, of course, the heat lost during the condensation process must be radiated away. Since TB is the main cooling process at very high temperature, one can write for the cooling time scale

$$
\tau=\frac{5 \mathrm{kT}^{1}}{2 \mathrm{Cn}}=8 \times 10^{3} \mathrm{~T}^{1 / 2} \mathrm{n}^{-1} \text { years }
$$


where we assume that the cooling occurs at constant pressure, $\mathrm{p} / \mathrm{k}=$ constant $=1100 \mathrm{~cm}^{-3} \mathrm{~K}$. For this particular value of the pressure,

$$
\tau=7 \mathrm{~T}^{3 / 2} \text { years }
$$

which is $9 \times 10^{12}$ years for $\mathrm{T}=4.4 \times 10^{8} \mathrm{~K}$, much longer than a Hubble time. Thus, radiation is unimportant in the IGM itself.

However, one can imagine a situation in which a cool dense region is confined by the hot IGM, and that thermal conduction carries heat from the IGM into the cool region, where it is radiated away. An outward temperature gradient would be set up, accompanied by an inward density gradient (because of the constant-pressure condition). In this case radiative cooling would become important at the point where $\tau \simeq \mathrm{H}^{-1}$, which from equation (8) requires $\mathrm{T} \sim 10 \mathrm{~K}, \mathrm{n} \sim 10^{-3}$ $\mathrm{cm}^{-3}$. These conditions are reminiscent of those in a galactic halo (Cowie, paper earlier in this Discussion).

The concept of a hot gas condensing into a radiatively cooled cloud has been applied in a somewhat different context by Gunn and Gott (1972) and by Mathews (1978), who propose that hot intracluster gas can accrete on to giant galaxies like M87 by this process, perhaps accounting for the activity observed in such galaxies. Cowie and Binney (1977) propose that the amount of intracluster gas may be regulated by this process, because gas will condense at the center of a cluster whenever its density is high enough that $\tau$ falls below $\mathrm{H}^{-1}$. It would be interesting to see whether significant modifications in the Cowie-Binney model would occur if, instead of negligible pressure at infinity, there were a pressure of the order of $10^{3} \mathrm{~cm}^{-3} \mathrm{~K}$ due to a hot IGM.

As was shown by Cowie and McKee (1976a), hot gas such as is being discussed here can also cause evaporation of cooler clouds embedded in it, provided the clouds are sufficiently small. They applied this (Cowie and McKee 1976b) to the HI clouds in Sculptor found by Mathewson et al. (1975), and argued that in fact such clouds would long ago have evaporated if they were exposed to a dense hot IGM, thus concluding that the latter does not exist. Further work by Haynes and Roberts (1979) suggests that the Sculptor clouds are actually part of our own galaxy, so the Cowie and McKee argument loses force. In fact, further searches of the type conducted by Mathewson et al. have failed to turn up any cases of true intergalactic clouds (Haynes and Roberts 1979, Lo and Sargent 1979).

The most significant effect of a dense hot IGM is on cosmology. The value $\Omega_{\text {a }}=0.5$ derived from the $\mathrm{x}$-ray background is somewhat uncertain, and a value close to $l$ is not excluded. However, as we have stated earlier, there are serious problems with any value of $\Omega$ greater than 0.1 , based on the abundance of ${ }^{2} \mathrm{H}$ and the difficulty of heating the IGM to the necessary temperature. From an observational point of view, we need careful studies of the degree to which the 
x-ray background can be accounted for by discrete sources, hopefully at higher energies than studied so far, and spacecraft measurements of possible distortions of the cosmic microwave background.

\section{REFERENCES}

Abe11, G.0.: 1965, Ann. Rev. Astron. Astrophys. 3, 1.

Cowie, L.L., Binney, J.: 1977, Ap.J. 215, 723.

Cowie, L.L., McKee, C.F.: 1976a, Ap.J. 211, 135.

Cowie, L.L., McKee, C.F.: 1976b, Ap.J. 209, L105.

Cowsik, R., Kobetich, E.J.: 1972, Ap.J. 177, 585.

Field, G.B.: 1979 (in preparation).

Fie1d, G.B., Perrenod, S.C.: 1977, Ap.J. 215, 717.

Giacconi, R.: 1979 (in preparation).

Giacconi, R., Bechtold, J., Branduardi, G., Forman, W., Henry, J.P., Jones, C., Kellogg, E., van der Laan, H., Liller, W., Marshaj.1, H., Murray, S.S., Pye, J., Schreier, E., Sargent, W.L.W., Seward, F., Tananbaum, H.: 1979, Ap.J., in press.

Gold, T., Hoyle, F.: 1959, "Paris Symposium on Radio Astronomy", ed. R.N. Bracewe11 (Stanford: Stanford University Press), paper 104 , p. 583.

Gott, J.R., Gunn, J.E., Schramm, D.N., Tinsley, B.M.: 1974, Ap.J. 194, 543.

Gunn, J.E., Gott, J.R. III: 1972, Ap.J. 176, 1.

Haynes, M.P., Roberts, M.S.: 1979, Ap.J. 227, 767.

Lo, K.Y., Sargent, W.L.W.: 1979, Ap.J. 227, 756.

Maraschi, L., Perola, G.C.: 1975, Astron. Astrophys. 40, 387.

Marsha11, F.E., Boldt, E.A., Holt, S.S., Miller, R., Mushotzky, R.F., Rose, L.A., Rothschild, R.E., Serlemitsos, P.J.: 1979, Preprint, Goddard Space Flight Center.

Mathews, W.G.: 1978, Ap.J., 219, 413.

Mathewson, D.S., Cleary, M.N., Murray, J.D.: 1975, Ap.J. 195, L97.

Schwartz, D.A.:1979, PROC. COSPAR-I.A.U. Symposium on X-Ray Astronomy, ed. L. Peterson and W. Baity (in press).

Wright, E.L.: 1979, Ap.J. (in press).

Yang, J., Schramm, D.N., Steigman, G., Rood, R.T.: 1979, Ap.J. 227, 697. 